\title{
SIMS on the Helium Ion Microscope: a Powerful Tool for High-Resolution High- Sensitivity Nano-Analytics
}

\author{
Tom Wirtz, David Dowsett, Jean-Nicolas Audinot, Santhana Eswara
}

Advanced Instrumentation for Ion Nano-Analytics (AINA), MRT Department, Luxembourg Institute of Science and Technology (LIST), 41 rue du Brill, L-4422 Belvaux, Luxembourg

Secondary Ion Mass Spectrometry (SIMS) is based on the detection of secondary ions emitted by a surface exposed to a primary ion bombardment. SIMS can be used to record mass spectra, images and depth profiles, providing excellent sensitivity, high dynamic range, high mass resolution and ability to differentiate between isotopes. As a consequence of recent instrument developments allowing very high lateral resolution in SIMS imaging, new fields of application, e.g. in life sciences, nanotechnologies and astronomy, are emerging [1]. In addition, the possibility to detect isotopes enables investigation techniques based on isotopic labelling that are of highest interest both in in materials and life sciences [1].

The combination of He/Ne microscopy and SIMS offers very interesting prospects for several reasons. First, while the ORION Helium Ion Microscope has become an ideal high resolution imaging and nanofabrication tool, its analysis capability is currently limited. SIMS can fill this gap and provide the missing analytical capabilities [2,3]. Second, SIMS performed with the finely focused $\mathrm{He}^{+}$or $\mathrm{Ne}^{+}$beam offers the possibility to obtain SIMS information limited only by the size of the probe-sample interaction $(\sim 10 \mathrm{~nm})$ [1,2]. Third, SIMS images obtained on the Helium Ion Microscope can be directly correlated with high resolution $(0.5 \mathrm{~nm})$ secondary electron images of the same zone [1]. In such a correlative approach, the typical trade-off between detection limits and minimum detectable feature size in the SIMS can be overcome. The settings for SIMS imaging can be chosen in order to reach excellent detection limits at the cost of poorer lateral resolution (by increasing the pixel/voxel sizes to several tens of $\mathrm{nm}$ ), while the very high resolution secondary electron images provide the required structural details.

The SIMS add-on system that we developed for the ORION Helium Ion Microscope consists of secondary ion extraction optics, post-accelerating transfer optics and a mass spectrometer (Figure 1). The extraction optics use a low extraction field in order to maintain a very small $\mathrm{He}^{+} / \mathrm{Ne}^{+}$probe size while maximizing the extraction efficiency [4]. A post acceleration stage is then used to accelerate the secondary ions to the required energy for the spectrometer and a system of transfer lenses transports the beam and focusses it onto the entrance plane of the spectrometer. The spectrometer is a compact highperformance double focusing magnetic sector mass spectrometer based on a modified Mattauch-Herzog configuration. To enhance the ionization yield of emitted secondary ions and hence to optimize the detection limits, the SIMS add-on system is equipped with an integrated reactive gas delivery system $[5,6]$.

In this presentation we will present fundamental aspects related to performing SIMS with $\mathrm{He}^{+}$and $\mathrm{Ne}^{+}$ ion beams (ultimate spatial resolution taking into account the characteristics of the collision cascades, ionization yields, sensitivity), instrumental aspects (design, characteristics) and applications (mass spectra (Figure 3), depth profiles, images (Figure 2), correlative approach). 


\section{References}

[1] T. Wirtz, P. Philipp, J.-N. Audinot, D. Dowsett, S. Eswara, Nanotechnology 26 (2015) 434001

[2] T. Wirtz, N. Vanhove, L. Pillatsch, D. Dowsett, S. Sijbrandij, J. Notte, Appl. Phys. Lett. 101 (2012) 041601

[3] L. Pillatsch, N. Vanhove, D. Dowsett, S. Sijbrandij, J. Notte, T. Wirtz, Appl. Surf. Sci. 282 (2013) 908

[4] D. Dowsett, T. Wirtz, N. Vanhove, L. Pillatsch, S. Sijbrandij, J. Notte, J. Vac. Sci. Technol. B 30 (2012) 06F602

[5] P. Philipp, T. Wirtz, H.-N. Migeon, H. Scherrer, Int. J. Mass. Spectrom. 253 (2006) 71

[6] T. Wirtz, H.-N. Migeon, Appl. Surf. Sci. 231-232 (2004) 940

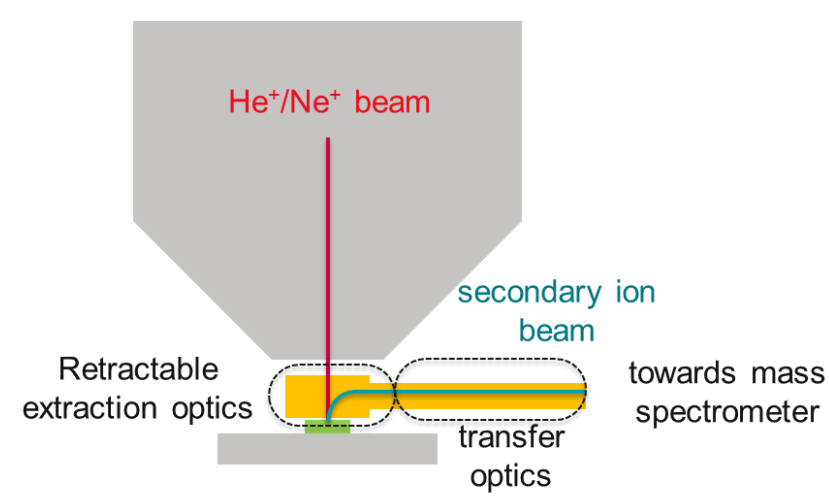

Figure 1: Schematic of the near sample region of the HIM with SIMS extraction optics.

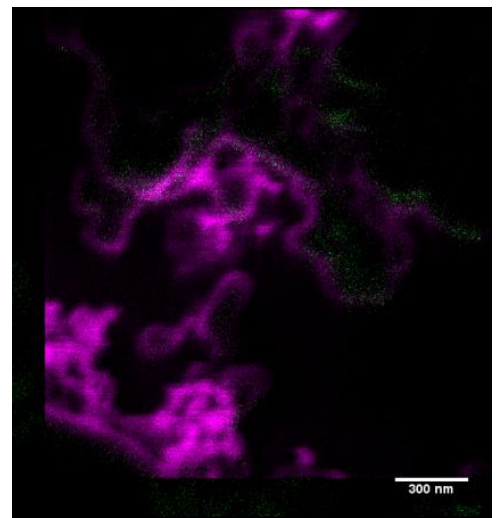

Figure 2: ${ }^{7} \mathrm{Li}$ (magenta) and ${ }^{11} \mathrm{~B}$ (green) SIMS image for a lithium titanate boron nitride nanoparticle mixture. The field of view is $1.9 \mu \mathrm{m}$. Primary beam $27 \mathrm{keV} \mathrm{Ne}^{+}$at $6 \mathrm{pA}$.

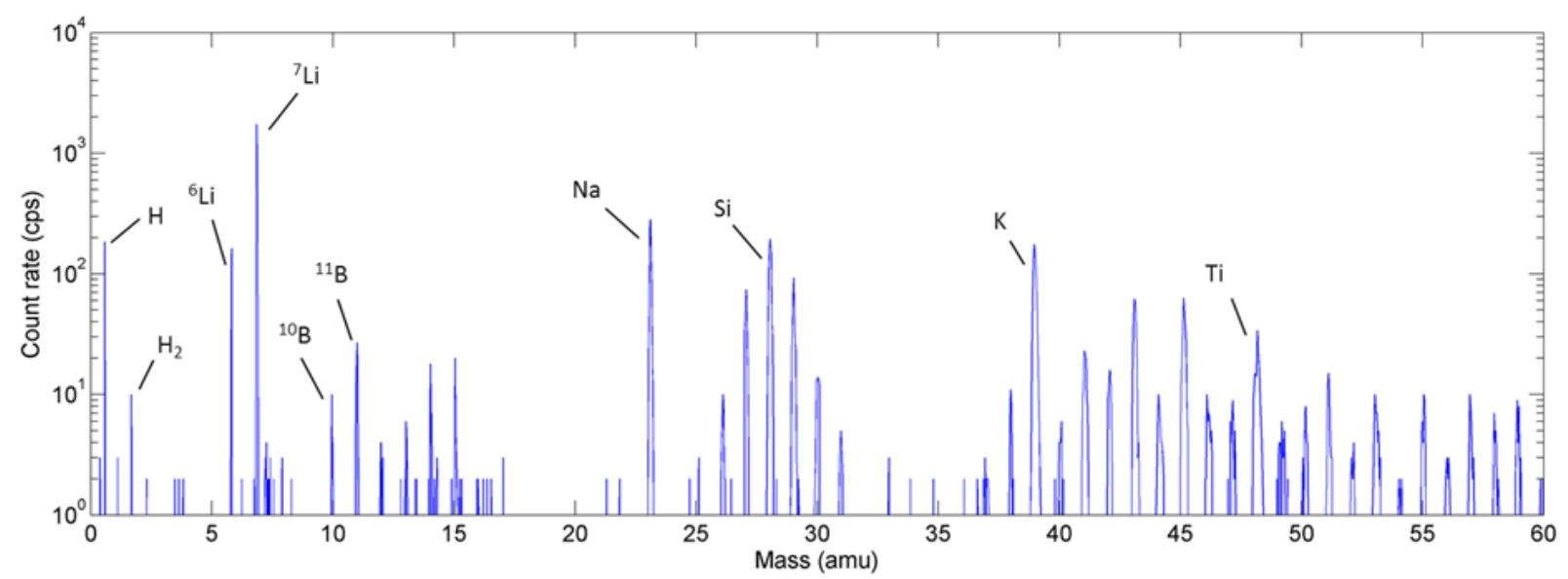

Figure 3: Mass spectrum of a lithium titanate and boron nitride nanoparticle mixture on a silicon substrate (primary beam $\mathrm{Ne}+25 \mathrm{keV}$ at $6 \mathrm{pA}$ ). 